\title{
Ensaios de campo para determinação de emissões de biogás em camadas de cobertura de aterros de resíduos sólidos
}

\section{Field tests for biogas emissions determination in cover layers of municipal solid waste landfills}

\author{
Maria Odete Holanda Mariano \\ Professora Adjunta do Núcleo de Tecnologia do Centro Acadêmico do Agreste da Universidade Federal de Pernambuco (UFPE). Doutora pela UFPE. \\ Pesquisadora do Grupo de Resíduos Sólidos da UFPE(GRS/UFPE)
}

José Fernando Thomé Jucá

Professor Associado do Departamento de Engenharia Civil da UFPE. Doutor pela Universidad Politécnica de Madrid, Espanha. Coordenador do GRS/UFPE.

\begin{abstract}
Resumo
Este trabalho apresenta uma metodologia de campo utilizada para determinar as emissões de biogás através das camadas de cobertura de aterros de resíduos sólidos urbanos, por meio de ensaios simples, diretos e de baixo custo. Os resultados obtidos nesses estudos indicaram que o fluxo de metano $\left(\mathrm{CH}_{4}\right)$ pode atingir valores de até $150 \mathrm{~kg} / \mathrm{m}^{2}$. ano, o que representa 630 mil toneladas do $\mathrm{CO}_{2}$ eq por ano em um simples aterro de 20 hectares. A espessura da camada de cobertura, que variou entre 0,2 e 0,7 m, não foi um fator relevante nas emissões medidas, enquanto que as pressões de biogás no contato camada-lixo, com valores acima de $500 \mathrm{~Pa}$, favorecem a formação de microfissuras no solo da camada de cobertura, o que eleva as emissões de gases nesses pontos.
\end{abstract}

Palavras-chave: aterro de resíduos sólidos urbanos; camadas de cobertura; fluxo de gases; ensaios de campo.

\section{Abstract}

This paper presents a field methodology used to determine emissions of biogas through the cover layers of municipal solid waste landfills, by means of simple, straightforward and inexpensive tests. The results from these studies indicated that the flow of methane $\left(\mathrm{CH}_{4}\right) \mathrm{can}$ reach values up to $150 \mathrm{~kg} / \mathrm{m}^{2}$. year, representing 630,000 tons of $\mathrm{CO}_{2}$ eq per year in a mere 20 hectares of landfill. The thickness of the cover layer, which ranged between 0.2 and $0.7 \mathrm{~m}$, was not a relevant factor in the measured emissions, while the pressure of biogas in the contact layer-waste, with values above $500 \mathrm{~Pa}$, favors the formation of microcracks on the soil of the cover layer, which increases the emissions of gases in these points.

Keywords: municipal solid waste landfill; top cover; gases flow; field tests.

\section{Introdução}

Os aterros de resíduos sólidos urbanos devem ser fundamentados em critérios de engenharia para evitar, além da poluição dos recursos hídricos e do solo, a poluição atmosférica. Assim, a emissão incontrolada do biogás é um grave problema de poluição atmosférica, tanto em nível local quanto em nível global e, consequentemente, sua emissão deve ser minimizada.

Nesse sentido, o sistema de cobertura dos resíduos é o principal elemento de projeto para evitar ou minimizar a poluição do ar devido aos gases gerados em aterros de resíduos sólidos, já que é o elo existente entre o ambiente interno dos resíduos e a atmosfera.
O conceito tradicional de sistema de cobertura de aterros de resíduos sólidos urbanos (ARSU) propõe que a camada funcione como uma barreira de impermeabilização dos resíduos frente às condições do ambiente externo, impedindo a infiltração da água precipitada e a liberação de gases para atmosfera. Assim, a camada de cobertura deve possuir uma série de características como, por exemplo, baixa permeabilidade à água e ao ar e durabilidade, ao longo do tempo.

Segundo Mariano (2008), estudos realizados por Albright et al. (2005), Montgomery e Parsons (1990), Melchior (1997) e Khire et al. (1997) mostram que as alterações das camadas de cobertura de argila compactada devido ao ciclo de umedecimento e secagem podem aumentar a condutividade hidráulica em torno de três ordens 
de grandeza, pois causam uma degradação mais rápida da camada de cobertura de solo e consequente perda de sua eficiência, em relação à condutividade hidráulica, em curto período de tempo.

Para a medição do fluxo de gases pelas camadas de cobertura, os principais métodos de investigação correspondem à placa de fluxo estática, placa de fluxo dinâmica e análises por infravermelho.

Em geral, as placas de fluxo são utilizadas para medições pontuais, em que caixas fechadas (estáticas) ou semiabertas (dinâmicas) são cravadas no solo, enquanto as análises por infravermelho são utilizadas para grandes áreas e para uma avaliação geral das emissões nas quais a concentração do biogás é determinada acima da superfície do aterro por processos óticos e/ou térmicos através da passagem de luz infravermelha (MARIANO, 2008).

A utilização de placas de fluxo apresenta vantagens por ser um método mais preciso para a determinação do fluxo pontual, exigir mão de obra pouco especializada, possibilitar a determinação das características e parâmetros do solo de cobertura no local do ensaio, permitir a avaliação simultânea de diversos gases, além de possibilitar a análise da influência da idade do resíduo, das condições atmosféricas e das pressões do gás no contato solo-resíduo (COSSU et al., 1997). Porém, possui como maior desvantagem a necessidade de realização de vários ensaios para a determinação da emissão total de um aterro.

Em aterros de resíduos sólidos, o gás mais estudado corresponde ao $\mathrm{CH}_{4}$, devido ao seu impacto ambiental causador do efeito estufa e à possibilidade de aproveitamento energético do mesmo.

Mariano (2008) apresenta 12 estudos sobre medição das emissões de $\mathrm{CH}_{4}$ em aterros de resíduos sólidos por meio de placa de fluxo estática, onde as taxas de emissões variam de 0,004 a $363 \mathrm{~g} /$ $\mathrm{m}^{2}$. dia. Maciel (2009) apresenta 23 estudos de emissões de $\mathrm{CH}_{4}$, nos quais essas taxas variam de $-0,29$ até $14.794 \mathrm{~g} / \mathrm{m}^{2}$. dia.

Essas variações de resultados comprovam que as emissões superficiais são dependentes de um conjunto de fatores, relacionados ao ambiente interno dos resíduos e à camada de cobertura, dependendo das características geotécnicas do solo, idade do resíduo, espessura da camada e o tipo de material utilizado, além do clima específico de cada aterro.

Nesse contexto, este trabalho apresenta a metodologia dos ensaios de campo para a determinação do fluxo de gases, espessura da camada de cobertura e pressões no contato solo-resíduo, bem como o mapeamento das emissões de $\mathrm{CH}_{4}$ na área estudada. O local de estudo foi o aterro de resíduos sólidos urbanos da cidade de Olinda, Pernambuco, e a metodologia adotada para avaliar as emissões de gases se mostrou um procedimento de baixo custo, fácil utilização e manuseio e que pode ser utilizado em qualquer aterro.

\section{Material e métodos}

Para a avaliação da emissão de gases pela camada de cobertura foi utilizado como local dos experimentos a camada de cobertura do aterro de resíduos sólidos de Aguazinha, Olinda, Pernambuco.
Os ensaios de campo realizados foram os ensaios de placa de fluxo estática, medição de pressão e concentração do biogás no contato solo-resíduo, determinação da espessura da camada de cobertura e a coleta amostras de solo para ensaios laboratoriais.

\section{Local de estudo}

O local utilizado para os estudos foi a Célula 1 do aterro de resíduos sólidos de Aguazinha, que se situa no Município de Olinda, Zona Norte da Região Metropolitana do Recife (RMR). O município de Olinda possui uma área de 40,83 km² e população de 368.666 habitantes (IBGE, 2000), e está inserido numa região de clima tropical litorâneo, quente e úmido, tipo Ams (segundo a classificação de Koppen), com uma precipitação média anual de $2.032 \mathrm{~mm}$.

$\mathrm{O}$ aterro de resíduos sólidos de Aguazinha possui uma área aproximada de 20 hectares. Sua operação foi iniciada em 1988 e, atualmente, recebe 400 toneladas/dia de resíduos sólidos urbanos. A espessura média de resíduo depositado é de $20 \mathrm{~m}$.

\section{Ensaios realizados}

As determinações dos fluxos dos gases $\mathrm{CH}_{4}$ através da camada de cobertura do aterro de Aguazinha foram realizadas utilizando-se a metodologia do ensaio com placa de fluxo estática em combinação com a medida de pressão e concentração dos gases.

Os ensaios foram realizados em toda a área da camada de cobertura da Célula 1, que possui um perímetro de $655 \mathrm{~m}$ e área de topo de $26.200 \mathrm{~m}^{2}$. No total foram realizados 19 ensaios de placa de fluxo e 19 ensaios com o dispositivo para medição de pressão e concentração do biogás no contato solo-resíduo, distribuídos de forma a cobrir toda área existente. Em todos os ensaios de placa de fluxo foram coletados solo para ensaios de laboratório.

Os equipamentos utilizados para medição de biogás foram Drager X-am 700, Dwyer ${ }^{\circledR} 477-2$, Dwyer ${ }^{\circledR}$ e Termometer Appa Mt-520, cujas características estão apresentadas na Tabela 1.

\section{Ensaio de placa de fluxo estática}

A placa para determinação dos fluxos de gases é construída em aço galvanizado com $2 \mathrm{~mm}$ de espessura. O topo de acrílico, com espessura de $8 \mathrm{~mm}$, é fixado na parte metálica. A placa tem formato de degrau para garantir que, ao ser cravada na camada de cobertura, evitará a entrada de ar atmosférico para o interior da caixa, além de facilitar a sua cravação no solo. O degrau fica em contato direto com o solo, de maneira que a área útil da placa seja a única área de passagem de fluxo de biogás pela camada de cobertura durante o ensaio. No topo da caixa existem duas conexões de saída (do tipo encaixe rápido), onde são conectados os equipamentos de medição de concentração e pressão dos gases. A placa também possui entradas para adaptação de equipamento para medição da temperatura do gás. 
Tabela 1 - Características dos equipamentos de medição do biogás

\begin{tabular}{|c|c|c|c|c|}
\hline Equipamentos & & Parâmetro & Faixa de medição & Faixa de erro do equipamento \\
\hline \multirow[t]{5}{*}{ Drager X-am 7000} & $\mathrm{CO}_{2}$ & \multirow[t]{5}{*}{ Concentração } & $0-100 \%$ & $\pm 2,0 \%$ \\
\hline & $\mathrm{CH}_{4}$ & & $0-100 \%$ & $\pm 5,0 \%$ \\
\hline & $\mathrm{H}_{2} \mathrm{~S}$ & & $0-500 \mathrm{ppm}$ & $\pm 5,0 \%$ \\
\hline & $\mathrm{O}_{2}$ & & $0-25 \%$ & $\pm 1,0 \%$ \\
\hline & $\mathrm{CO}$ & & $0-500$ ppm & $\pm 1,0 \%$ \\
\hline Dwyer $^{\circledR} 477-2$ & & Pressão & $0-10,0 \mathrm{kPa}$ & $\pm 1,0 \%$ \\
\hline Dwyer $^{\circledR}$ & & Velocidade do ar & $0-7 \mathrm{~m} / \mathrm{s}$ & $\pm 3,0 \%$ \\
\hline Termometer Appa Mt-520 & & Temperatura & -50 a $1.300^{\circ} \mathrm{C}$ & $\pm 0,5 \%$ \\
\hline
\end{tabular}
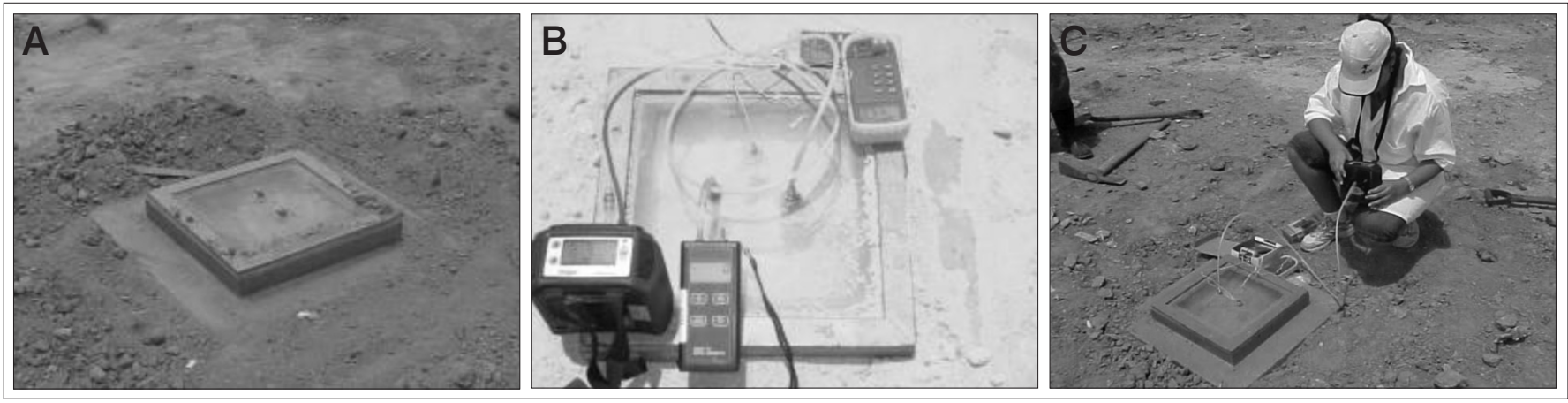

Figura 1 - Instalação e leitura do ensaio de placa de fluxo. A) Cobertura da placa; B) equipamentos utilizados; C) leitura da concentração dos gases

A instalação da placa de fluxo seguiu o seguinte procedimento:

- inicialmente, foi colocada a placa no local do ensaio e demarcado seu perímetro $(0,60 \mathrm{~m} \times 0,60 \mathrm{~m})$;

- retira-se a placa do local e marca-se 0,10 m de cada lado como folga - essa nova área é demarcada;

- escava-se uma pequena vala de, no máximo, 0,15 m para a gravação da placa;

- após essa escavação, coloca-se novamente a placa no local e "talham-se" cuidadosamente as paredes laterais do quadrado para que o mesmo fique com as dimensões externas da placa $(0,60 \mathrm{~m}$ x $0,60 \mathrm{~m}$ );

- pressiona-se a caixa no solo de forma que a cravação seja vertical com o conector de saída aberto, evitando o acúmulo de gases no interior da placa;

- após a cravação da placa, o solo do seu entorno é recolocado na vala e compactado manualmente.

A Figura 1 apresenta o processo de instalação da placa de fluxo.

A leitura da concentração dos gases na placa de fluxo estática é feita logo após a sua cravação, e esse momento foi considerado o instante inicial de cada ensaio. O tempo de duração total do ensaio foi de 180 minutos. Adotou-se como intervalo de leitura do ensaio 1 minuto para os primeiros 5 minutos do ensaio; depois, um intervalo de 5 minutos para a primeira hora do ensaio, intervalo de 10 até 120 minutos de ensaio e intervalos de 15 até 180 minutos. O procedimento de leitura da concentração do biogás consiste em:

- acoplar o equipamento de leitura da concentração do gás;

- abertura da mangueira de polietileno para início da leitura;

- tempo de estabilização de 2 minutos;

- leitura das concentrações;

- fechamento da mangueira de polietileno para finalização da leitura;

- retirada do equipamento.

\section{Medição de pressão e concentração do biogás}

A localização dos ensaios para a medição da pressão e concentração dos gases no contato solo-resíduo é feita utilizando-se o dispositivo de medição de pressão e concentração, que deve ser instalado próximo ao local onde está sendo realizado o ensaio de placa de fluxo. Esse equipamento foi instalado a uma distância de, aproximadamente, $2 \mathrm{~m}$ da placa de fluxo estática.

Os equipamentos do dispositivo de medição de pressão e concentração são fabricados com tubos e CAP's de PCV com diâmetro de $0,127 \mathrm{~m}$ (5 polegadas) e comprimento variável. O CAP de PVC possui uma saída para conexão com o equipamento de medição das concentrações dos gases e leituras da pressão. Inicialmente, a camada de cobertura é escavada com um amostrador do tipo trado manual $(\bigotimes=5$ ") até o contato da camada com o resíduo depositado. 
O procedimento para cravação do dispositivo de medição de pressão e concentração do biogás no contato solo-resíduo e leituras é descrito a seguir:

- perfuração com trado manual da camada de cobertura até encontrar o contato solo-resíduo;

- cravação do tubo PVC até a profundidade do contato soloresíduo;

- a saída do CAP é fechada para que não ocorra um alívio de pressões e concentração. No momento da leitura, os equipamentos de pressão e concentração dos gases são acoplados e, então, a saída do CAP de PVC é aberta para que se possa efetuar a leitura;

- posteriormente, a saída do CAP é fechada e os equipamentos, retirados;

- as leituras são realizadas em intervalos de 20 a 30 minutos durante todo o tempo de duração do ensaio;

- após a finalização do ensaio, o tubo de PVC é retirado e medida a espessura da camada de cobertura com escala com precisão de $1 \mathrm{~mm}$. A Figura 2 apresenta o procedimento de leituras no CAP.

\section{Determinação do fluxo de gases}

A partir dos dados obtidos em cada ensaio de placa de fluxo estática, determinou-se o fluxo mássico de gás, conforme a Equação 1 (CZEPIEL et al, 1996):

Fluxo $=\frac{V_{p} \rho_{g a \dot{s} s}}{A} \frac{\Delta C}{\Delta t}$ Equação 1

onde:

$\mathrm{V}_{\mathrm{p}}$ : Volume da placa $=0,008 \mathrm{~m}^{3}$;

A: Área da Placa $=0,16 \mathrm{~m}^{2}$;

$\rho_{\text {gás }}$ : densidade do gás a uma dada temperatura $\left(\mathrm{kg} / \mathrm{m}^{3}\right)$;

$\Delta \mathrm{C} / \Delta \mathrm{t}$ : variação da concentração do gás (\% volume) com o tempo (segundos).

Para calcular o fluxo, utilizam-se as curvas de variação de concentração do gás com o tempo sejam representadas em termos mássi$\cos (\Delta \mathrm{M} / \Delta \mathrm{t})$. Nesse sentido, utiliza-se a densidade corrigida dos gases
$\left(\mathrm{CH}_{4}\right.$ e $\left.\mathrm{CO}_{2}\right)$ em função das leituras das temperaturas internas durante o ensaio de placa de fluxo considerando que (Equações 2 e 3):

$\rho_{\mathrm{CH}_{+}}(t)=\frac{\rho_{\mathrm{CH}_{4}}\left(0^{\circ} \mathrm{C}\right) \times 273}{\left[273+t\left({ }^{\circ} \mathrm{C}\right)\right]}$

Equação 2

$\rho_{\mathrm{CO}_{2}}(t)=\frac{\rho_{\mathrm{CH}_{+}}\left(t^{\circ} \mathrm{C}\right) \times \mathrm{PM}_{\mathrm{CO}_{2}}}{P M_{\mathrm{CH}_{+}}}$

Equação 3

onde:

$\mathrm{\rho CH}_{4}(\mathrm{t})$ : densidade do $\mathrm{CH}_{4}$ em função da temperatura $\left({ }^{\circ} \mathrm{C}\right)$;

$\rho \mathrm{CH}_{4}: 0,716 \mathrm{~kg} / \mathrm{m}^{3}$;

$\rho \mathrm{CO}_{2}(\mathrm{t})$ : densidade do $\mathrm{CO}_{2}$ em função da temperatura $\left({ }^{\circ} \mathrm{C}\right)$;

$\mathrm{PM} \mathrm{CO}_{2}$ : peso molecular do $\mathrm{CO}_{2}=44$;

$\mathrm{PM} \mathrm{CH}_{4}$ : peso molecular do $\mathrm{CH}_{4}=16$.

O fluxo mássico foi calculado no intervalo inicial do ensaio para se obter a maior taxa de percolação do gás pela camada de cobertura, simulando a condição do aterro de resíduos, onde a camada de cobertura fica em contato com a atmosfera e, consequentemente, ocorrem os gradientes máximos de pressão e concentração.

$\mathrm{O}$ procedimento para a determinação do fluxo em cada ensaio foi o seguinte:

- correção da densidade do gás em função da temperatura no instante " $\mathrm{t}$ " do ensaio;

- cálculo da massa do gás para cada tempo "t" do ensaio;

- construção do gráfico Massa [M.L-2] x Tempo [T];

- determinação da equação para o fluxo, considerando o tempo $t_{\text {máx }}$ $=60$ minutos:

- determinação da equação de regressão com coeficiente de determinação $\geq 95 \%$;

- determinação do fluxo para um ano.

A Figura 3 apresenta o esquema adotado para o cálculo do fluxo.

\section{Resultados e discussão}

A Tabela 2 apresenta o resumo dos principais resultados obtidos nos ensaios de campo em ordem crescente de fluxo de $\mathrm{CH}_{4}$. A Tabela
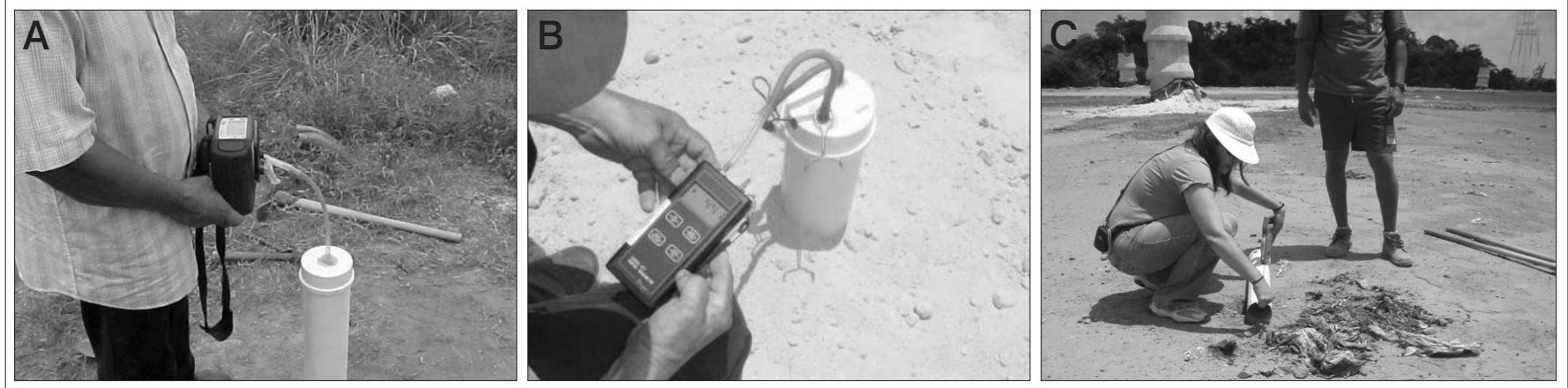

Figura 2 - Instalação e leitura da concentração dos gases e pressão no contato solo-resíduo e medição da espessura da camada. A) Leitura da concentração dos gases; B) leitura da pressão; C) medição da espessura da camada 
2 apresenta os principais resultados obtidos nos ensaios de campo realizados referente ao fluxo medido, espessura da camada, concentração e pressão dos gases no contato solo-resíduo. Também foram calculados o percentual de retenção de $\mathrm{CH}_{4}, \mathrm{CO}_{2}$ e $\mathrm{H}_{2} \mathrm{~S}$ pela camada de cobertura. Para obtenção das planilhas de cada ensaio realizado de forma mais detalhada, ver Mariano (2008).

A ampla faixa de variação do fluxo e $\mathrm{CH}_{4}\left(0\right.$ a $146 \mathrm{~kg} / \mathrm{m}^{2}$. ano $)$ indica que existem vários fatores que interferem na emissão dos gases pela camada de cobertura, que vão desde as características geotécnicas do solo (tipo, grau de compactação e de saturação), qualidade e espessura da camada de cobertura, pressões de gases no contato solo-resíduo, idade dos resíduos, condições climáticas da região, entre outros (MARIANO, 2008). Esses valores indicaram que as emissões de gases pela camada de cobertura representaram 22\% do total das emissões de $\mathrm{CH}_{4}$ do aterro.

A distribuição irregular e aleatória da espessura da camada $(0,24$ a 0,69 m) indica que a mesma não apresenta nenhuma relação direta com as emissões de $\mathrm{CH}_{4}$ pela camada de cobertura. Esse fato, apesar de surpreendente, mostra a importância do estudo das características e parâmetros do solo, bem como a avaliação das condições em campo. Esses resultados também indicam que a espessura do solo por si só pode ser considerado um fator secundário da emissão dos gases pela camada.

Observa-se também que o percentual de retenção de $\mathrm{CH}_{4}$ pela camada de cobertura é maior que o percentual de retenção de $\mathrm{CO}_{2}$.

Tabela 2 - Resumo dos principais resultados dos ensaios de campo

\begin{tabular}{|c|c|c|c|c|c|c|c|}
\hline \multirow{2}{*}{ Ensaio } & \multirow{2}{*}{$\begin{array}{c}\text { Espessura da } \\
\text { camada }(\mathrm{m})\end{array}$} & \multirow{2}{*}{$\begin{array}{l}\text { Pressão contato } \\
\text { solo-resíduo }\end{array}$} & \multirow{2}{*}{$\begin{array}{l}\text { Concentração de } \\
\mathrm{O}_{2}(\%) \text { contato } \\
\text { solo-resíduo }\end{array}$} & \multirow{2}{*}{$\begin{array}{l}\text { Fluxo de } \mathrm{CH}_{4} \\
\text { pela camada } \\
\left(\mathrm{kg} / \mathrm{m}^{2} \text {.ano) }\right.\end{array}$} & \multicolumn{3}{|c|}{ \% de Retenção dos gases pela camada de cobertura } \\
\hline & & & & & $\mathrm{CO}_{2}$ & $\mathrm{CH}_{4}$ & $\mathrm{H}_{2} \mathrm{~S}$ \\
\hline A 2 & 0,33 & 10 & $3 \%$ & 0 & 90 & 99 & 100 \\
\hline A 12 & 0,38 & 87 & $0 \%$ & 0 & 98 & 99 & 100 \\
\hline A 13 & 0,36 & -84 & $1 \%$ & 0 & 98 & 99 & 100 \\
\hline A 15 & 0,41 & 3.830 & $9 \%$ & 0 & 96 & 100 & 100 \\
\hline A 10 & 0,60 & 11 & $2 \%$ & 7 & 77 & 90 & - \\
\hline A 3 & 0,24 & 16 & $3 \%$ & 14 & 66 & 81 & 100 \\
\hline A 4 & 0,30 & 25 & $3 \%$ & 14 & 76 & 87 & 100 \\
\hline A 18 & 0,39 & 16 & $12 \%$ & 15 & 47 & 80 & 100 \\
\hline A 20 & 0,54 & 16 & $7 \%$ & 15 & 65 & 81 & - \\
\hline A 1 & 0,60 & 12 & $10 \%$ & 24 & 69 & 80 & - \\
\hline A 14 & 0,48 & 8 & $15 \%$ & 25 & 35 & 58 & 100 \\
\hline A 21 & 0,54 & 16 & $7 \%$ & 26 & 47 & 74 & - \\
\hline A 6 & 0,52 & 208 & $0 \%$ & 31 & 70 & 77 & 100 \\
\hline A 19 & 0,39 & 16 & $12 \%$ & 32 & 35 & 38 & 40 \\
\hline A 11 & 0,69 & 6 & $1 \%$ & 44 & 68 & 75 & 100 \\
\hline A 8 & 0,40 & 12 & $1 \%$ & 49 & 3 & 0 & 100 \\
\hline A 7 & 0,61 & 560 & $0 \%$ & 56 & 42 & 56 & 100 \\
\hline A 5 & 0,60 & 901 & $2 \%$ & 70 & 40 & 43 & 100 \\
\hline A 9 & 0,40 & 2.230 & $0 \%$ & 146 & 0 & 10 & 100 \\
\hline
\end{tabular}




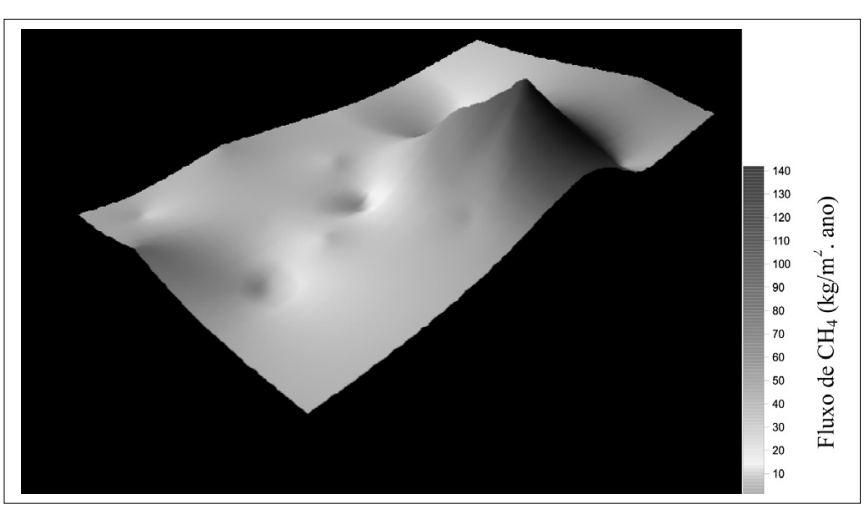

Figura 4 - Imagem tridimensional do fluxo de $\mathrm{CH}_{4}\left(\mathrm{~kg} / \mathrm{m}^{2}\right.$. ano)

na camada de cobertura do aterro de resíduos sólidos da Muribeca, Pernambuco.

$\mathrm{O}$ mapeamento das emissões de $\mathrm{CH}_{4}$ foi realizado por meio da técnica de interpolação de dados com o software Surfer ${ }^{\circledR} 8$ (Golden Software, Inc.) e a interpolação Kriggiana, utilizando-se todos os ensaios realizados na cota final do aterro. A Figura 4 apresenta a imagem tridimensional das emissões de $\mathrm{CH}_{4}$ pela camada de cobertura do aterro.

As áreas de maior valor de fluxo de $\mathrm{CH}_{4}$ pela camada de cobertura encontram-se nas extremidades do aterro, diminuindo no sentido do centro da Célula $n^{\circ} 1$, enquanto que as áreas de menor fluxo de $\mathrm{CH}_{4}$ encontram-se no centro do aterro, formando uma região de menor emissão de biogás. Os ensaios que representam essa faixa de fluxo correspondem à área de maior tráfego de equipamentos (carros e caminhões) na Célula (MARIANO, 2008).

\section{Conclusões}

Com base nos resultados obtidos neste estudo, pode-se concluir que:

- o ensaio de placa de fluxo mostrou-se um procedimento de fácil utilização e manuseio para a determinação das emissões dos gases pela camada de cobertura;

- o dispositivo de medição de pressão e concentração de gás no contato solo-resíduo mostrou-se um procedimento de fácil utilização;

- a medição da espessura da camada indicou uma distribuição irregular e aleatória, comprovando que a espessura do solo por si só pode ser considerado um fator secundário da emissão dos gases pela camada;

- o mapeamento das emissões de $\mathrm{CH}_{4}$ pela camada de cobertura mostrou-se uma ferramenta muito útil para a extrapolação dos resultados pontuais obtidos nos ensaios de placa de fluxo, com melhor visualização do comportamento na camada de cobertura do aterro, observando-se menores fluxos no centro da Célula e maiores nos bordos da Célula.

\section{Referências}

COSSU, R. et al. Biogás emission measurements using static and dynamic flux chambers and infrad methods. Proceedings of The Sixth International Landfill Symposium, v. 4, p. 103-114, 1997.

CZEPIEL, P.M. et al. Quantifying the effect of oxidation on landfill methane emissions. Journal of Geophysical Research, v. 101, n. D11, p. 16.721$16.729,1996$

INSTITUTO BRASILEIRO DE GEOGRAFIA E ESTATÍSTICA (IBGE). 2000. Contagem populacional. Disponível em <www.ibge.gov.br>. Acesso em: jul. 2008.
MACIEL, F.J. Geração de biogás e energia em aterro experimental de resíduos sólidos urbanos. Tese (Doutorado em Engenharia Civil) Universidade Federal de Pernambuco, Recife, PE, 2009.

. Estudo da Geração, Percolação e Emissão de Gases no Aterro de Resíduos Sólidos da Muribeca/PE. Dissertação (Mestrado em Engenharia Civil) - Universidade Federal de Pernambuco, Recife, PE, 2003.

MARIANO, M.O.H. Avaliação da retenção de gases em camadas de cobertura de aterros de resíduos sólidos. Tese (Doutorado em Engenharia Civil) - Universidade Federal de Pernambuco, Recife, PE, 2008. 